\title{
DESEMPENHO DE MOTOR DIESEL COM MISTURAS DE BIODIESEL DE ÓLEO DE GIRASSOL
}

\author{
Performance of diesel engine fuelled with sunflower biodiesel blends \\ Ila Maria Corrêa', José Valdemar Gonzalez Maziero ${ }^{2}$, Maria Regina Úngaro ${ }^{3}$, \\ José Augusto Bernardi ${ }^{4}$, Moises Storino
}

\begin{abstract}
RESUMO
Objetivou-se, neste trabalho avaliar o uso de misturas de biodiesel de girassol (Helianthus annuus L.) e diesel no desempenho de um motor de ignição por compressão, injeção direta. Os ensaios foram realizados em bancada dinamométrica utilizando-se as misturas B5, B10, B20 e B100 em comparação ao diesel (D). Foi analisado o desempenho do motor através da tomada de potência (TDP) com cada combustível, e analisado o óleo lubrificante do motor antes e após 96 horas de uso com B100. Os resultados obtidos foram: D (40,7 kW; 271 g/kW.h); B5 (40,3 kW; 271 g/kW.h); B10 (39,8 kW; 277 g/kW.h); B20 (40,0 kW; 277 g/kW.h) e B100 (39,8 kW; 291 g/kW.h). Concluiu-se que o uso das misturas B5, B10, B20 e B100 proporcionou redução de no máximo 2,2 \% na potência na TDP e um aumento máximo de 7,3\%, no consumo específico de combustível. A análise do óleo lubrificante, antes e após o uso com B100, detectou alterações aceitáveis, sendo a viscosidade, a presença de água e o teor de ferro os parâmetros mais expressivamente alterados.
\end{abstract}

Termos para indexação: Motor de compressão, desempenho, biocombustível.

\section{ABSTRACT}

This work aimed to evaluate the use of sunflower biodiesel (Helianthus annuus L.) blends in a CI engine, direct injection. The test procedure was carried out in a dynamometer bench that determined the performance of engine through power take-off (PTO) with use of diesel and sunflower biodiesel blends (B5, B10, B20 and B100). The lubricating oil was analyzed before and after period of 96 hours. The results were: D (40,7 kW; $271 \mathrm{~g} / \mathrm{kW} . h)$; B5 (40,3 kW; $271 \mathrm{~g} / \mathrm{kW} . \mathrm{h}) ;$ B10 (39,8 kW; $277 \mathrm{~g} / \mathrm{kW} . \mathrm{h}) ;$ B20 (40,0 kW; 277 $\mathrm{g} / \mathrm{kW} . \mathrm{h})$ e B100 (39,8 kW; $291 \mathrm{~g} / \mathrm{kW} . \mathrm{h})$. One conclude that the use of blends B5, B10, B20 and B100 decreased the power of PTO max. $2,2 \%$ and increased the fuel consumption max. 7, 3\%. The analysis of the lubricating oil before and after the use of B100 showed acceptable alterations and the viscosity, water content and level of iron were the most affected parameters.

Index terms: Engine compression, performance, biofuel.

(Recebido em 28 de abril de 2006 e aprovado em 21 de março de 2007)

\section{INTRODUÇÃO}

A substituição do óleo diesel por biocombustíveis ou misturas desse com diesel é a questão mais focada atualmente para suprir a escassez dos combustíveis derivados do petróleo e reduzir os níveis de emissão de poluentes gasosos. A autorização do uso de misturas com $2 \%$ de biodiesel (B2) pelo governo federal brasileiro é o passo inicial para reduzir o uso de óleo diesel e desencadear ações de pesquisa sobre óleos vegetais.

Em vista das inúmeras possibilidades de obtenção de matéria-prima (soja, mamona, girassol, palma, etc.) para a produção de biodiesel, o Brasil destaca-se no panorama mundial como potencial fornecedor de biodiesel. Qualquer que seja, porém, a matéria-prima utilizada é importante estudar suas características físico-químicas e seu efeito no desempenho mecânico do motor.

O estudo do uso de doze combustíveis alternativos, produzidos pela mistura de óleos vegetais com óleo diesel tipo 2, em um motor diesel, realizado por Ali et al. (1996), em bancada dinamométrica, mostrou que o desempenho do motor foi similar ao obtido com óleo diesel indicando que não haveria efeito no desempenho do motor após 200 horas do funcionamento no dinamômetro.

Para confirmar isso, Ali \& Hanna (1996) estudaram o efeito a longo prazo de uma mistura de óleo diesel e

\footnotetext{
Engenheira Agrícola, Doutora - Centro de Engenharia e Automação/CEA - Instituto Agronômico de Campinas/IAC - Rodovia D. Gabriel P. B. Couto, Km 65 - Aeroporto - Cx. P. 26 - 13201-970 - Jundiaí, SP - imcorrea@iac.sp.gov.br

${ }^{2}$ Engenheiro Agrônomo, Mestre - Centro de Engenharia e Automação/CEA - Instituto Agronômico de Campinas/IAC - Rodovia D. Gabriel P. B. Couto Km 65 - Aeroporto - Cx. P. 26 - 13201-970 - Jundiaí, SP -maziero@iac.sp.gov.br

${ }^{3}$ Engenheira Agrônoma, Doutora - Centro APTA de Grãos e Fibras - Instituto Agronômico de Campinas/IAC - Avenida Barão de Itapura, 1481 - Cx. P. 28 13001-970 - Campinas, SP - ungaro@iac.sp.gov.br

${ }^{4}$ Engenheiro Agrônomo - Centro de Engenharia e Automação/CEA - Instituto Agronômico de Campinas/IAC - Rodovia D. Gabriel P. B. Couto, Km 65 Aeroporto - Cx. P. 26 - 13201-970 - Jundiaí, SP -bernardi@iac.sp.gov.br

${ }^{5}$ Engenheiro Agrônomo, Mestre - Centro de Engenharia e Automação/CEA - Instituto Agronômico de Campinas/IAC - Rodovia D. Gabriel P. B. Couto, Km 65 - Aeroporto - Cx. P. 26 - 13201-970 - Jundiaí, SP - storino@iac.sp.gov.br
} 
biodiesel (B20) em relação ao desempenho e desgaste de um motor diesel. Constataram que o motor operou satisfatoriamente por 148 horas, e a potência, o torque e o consumo específico de combustível mantiveram-se constantes. A análise do óleo do motor a intervalos de 45 horas, mostrou que a redução na necessidade após 100 horas de operação, foi típica de uma operação normal com diesel. $\mathrm{O}$ desgaste do motor, avaliado com base na concentração de metais no óleo lubrificante, permaneceu dentro do limite normal recomendado para aquele tipo de motor.

A condução de trabalhos nessa linha de pesquisa auxiliará a identificar e solucionar problemas que possam vir a ocorrer em decorrência da utilização, breve ou prolongada, de maiores níveis de misturas de óleos vegetais ao óleo diesel. Objetivou-se no trabalho avaliar o desempenho de um motor de ignição, por compressão, utilizado em trator agrícola, ao ser alimentado com diferentes misturas de éter etílico de óleo de girassol (EEOG) e diesel, em ensaios realizados em bancada dinamométrica.

\section{MATERIAL E MÉTODOS}

\section{Combustíveis avaliados}

As misturas B5, B10, B20 e B100 de éter etílico de óleo de girassol foram avaliadas em comparação ao uso de óleo diesel (D). Os cinco tipos de combustíveis foram estudados em um motor MWM D229.3, injeção direta, com potência nominal de $46 \mathrm{~kW}$ à $2450 \mathrm{r} / \mathrm{min}$, no Laboratório de Dinamometria do Centro de Engenharia e Automação do Instituto Agronômico. O laboratório dispõe de um dinamômetro Schenck W 400 com capacidade para 400 $\mathrm{kW}$ e de um conjunto de sensores eletrônicos com os quais foram monitorados alguns pontos de temperatura do motor (entrada e saída de água, ar de admissão, óleo lubrificante e tubulação dos gases de escape).

O diesel utilizado foi adquirido em posto de abastecimento automotivo. A transesterificação etílica do óleo de girassol foi realizada pela empresa CERALIT SA. Ind. e Com. e suas características físico-químicas foram determinadas pelo Instituto de Pesquisas Tecnológicas. A densidade dos combustíveis foi determinada conforme descrito em Corrêa (2000). No Laboratório de Dinamometria do Centro de Engenharia e Automação, o sistema de determinação do consumo de combustível, do tipo volumétrico, era constituído de balão volumétrico, fotocélulas e eletro válvulas interligados, cronômetro e totalizador do número de giros dados pelo motor.

\section{Ensaios realizados}

Por se tratar de motor novo, adquirido especialmente para este trabalho, foi necessário realizar o amaciamento do motor, o que foi feito em bancada dinamométrica. O amaciamento durou 50 horas obedecendo a um regime de cargas variáveis, utilizando óleo diesel como combustível.

Para avaliação do desempenho do motor com os diferentes combustíveis foram realizados ensaios de curta duração (2 horas), com o motor operando à plena carga, isto é, em aceleração máxima da bomba injetora, porém, na condição de rotação nominal do motor. Durante as duas horas de ensaio, leituras de torque, rotação do motor, consumo de combustível e de temperaturas foram feitas a intervalos de 12 minutos. Este padrão de ensaio seguiu a norma ABNT (1995).

Após os ensaios de curta duração, o motor foi submetido a um regime de funcionamento de 96 horas (12 horas/dia), sob cargas variáveis, utilizando éster etílico de óleo de girassol (B100), sendo determinados, nesse período, os valores de potência e de consumo de combustível. Amostras de óleo lubrificante do motor foram tomadas para análise antes e após as 96 horas.

\section{Análise do óleo lubrificante}

Com a finalidade de verificar possíveis alterações nas características do óleo lubrificante, decorrentes do uso semi-prolongado (96 horas) do éster etílico de girassol (B100) foram determinadas as suas características físicoquímicas, teor de metais e exame morfológico das partículas contaminantes. A análise do teor de contaminação do óleo lubrificante, segundo Peterson et al. (1999), fornece uma excelente indicação das condições internas do motor. As análises foram realizadas pela empresa SIL - Serviços Industriais de Lubrificação.

\section{RESULTADOS E DISCUSSÃO}

O resultado da análise físico-química do éster etílico do óleo de girassol observa-se no Quadro 1, demonstrando que suas características estão dentro dos limites estabelecidos na Portaria ANP 255/03.

Os resultados dos ensaios de duas horas à rotação nominal do motor são apresentados no Quadro 2. Reduções estatisticamente significativas foram obtidas para a potência no motor com os diferentes combustíveis, porém, as diferenças porcentuais foram, no máximo, de - 2,2\%.

A rotação do motor, não apresentando diferenças significativas, demonstra que a condição de ensaio foi constante para todos os combustíveis estudados. Assim, as diferenças nas potências obtidas podem ser atribuídas às diferenças nos torques gerados como conseqüência provável do menor poder calorífico de cada mistura. Este, sendo inferior ao do diesel, era de se esperar alguma queda na potência do motor. 
Quadro 1-Características físico-químicas do EEOG utilizado no ensaio.

\begin{tabular}{|lll|}
\hline \multicolumn{1}{|c|}{ Característica } & \multicolumn{1}{c|}{ Material } & \multicolumn{1}{c|}{ Portaria ANP255/03 } \\
\hline Aspecto & Límpido e isento de impurezas & Límpido e isento de impurezas \\
Água e sedimentos, $\%$ em volume & $<0,05$ & máx: 0,05 \\
Cinzas sulfatadas, $\%$ & $<0,01$ & máx: 0,020 \\
Corrosão, 3h a $50^{\circ} \mathrm{C}$ & 1 & máx.: 1 \\
Massa específica a $20^{\circ} \mathrm{C}-\mathrm{kg} / \mathrm{m}^{3}$ & $879,2 \pm 0,1$ & anotar \\
Enxofre total $\%$ & $0,007 \pm 0,003$ & máx.: 0,20 \\
Índice de iodo, $\mathrm{g} / 100 \mathrm{~g}$ & $129,3 \pm 0,2$ & anotar \\
Número de cetano & 48,52 & min.: 45 \\
Ponto de fulgor, ${ }^{\circ} \mathrm{C}$ & resultados encontrados 92, & min.: 100 \\
& $106,115,119 \mathrm{e}>130$ & \\
Resíduo de carbono, $\%$ & 0,03 & máx.: 0,05 \\
Ponto de entupimento, ${ }^{\circ} \mathrm{C}$ & 2 & máx.: 9 \\
Teor de etanol & $0,062 \pm 0,007$ & máx, $: 0,5$ \\
Viscosidade cinemática a $40^{\circ} \mathrm{C}, \mathrm{mm}^{2} / \mathrm{s}$ & $4,61 \pm 0,02$ & 2,5 a 5,5 \\
Análise química & & Na $+\mathrm{K}$-max.: $10 \mathrm{mg} / \mathrm{kg}$ \\
$\quad$ Sódio & $1,5 \pm 0,4$ & máx.: $10 \mathrm{mg} / \mathrm{kg}$ \\
Potássio & $<1$ & \\
Fósforo & $<5$ & \\
\hline
\end{tabular}

Quadro 2 - Desempenho do motor à rotação nominal.

\begin{tabular}{|c|c|c|c|c|c|c|}
\hline \multirow[t]{2}{*}{ Combustível } & \multicolumn{2}{|c|}{ Rotação do Motor } & \multicolumn{2}{|c|}{ Potência Corrigida $^{1}$} & \multicolumn{2}{|c|}{ Consumo Específico } \\
\hline & Média $^{2}$ & D. Padrão & Média $^{2}$ & D. Padrão & Média $^{2}$ & D. Padrão \\
\hline & \multicolumn{2}{|c|}{$\mathrm{r} / \mathrm{min}$} & \multicolumn{2}{|c|}{$\mathrm{kw}$} & \multicolumn{2}{|c|}{$\mathrm{g} / \mathrm{kw} \cdot \mathrm{h}$} \\
\hline $\mathrm{D}$ & $2449 a$ & 11 & $40,7 a$ & 0,2 & $271 b$ & 1 \\
\hline B5 & $2448 a$ & 11 & $40,3 b$ & 0,2 & $271 b$ & 1 \\
\hline B10 & $2443 a$ & 8 & $39,8 c$ & 0,2 & $277 c$ & 2 \\
\hline B20 & $2452 a$ & 11 & $40,0 c$ & 0,3 & $277 c$ & 1 \\
\hline B100 & $2449 a$ & 9 & $39,8 c$ & 0,2 & $291 a$ & 1 \\
\hline Média & \multicolumn{2}{|c|}{2448} & \multicolumn{2}{|c|}{40,1} & \multicolumn{2}{|c|}{278} \\
\hline C.V.,\% & \multicolumn{2}{|c|}{0,42} & \multicolumn{2}{|c|}{0,55} & \multicolumn{2}{|c|}{0,50} \\
\hline
\end{tabular}

${ }^{1}$ Potência corrigida pela norma ABNT (1996).

${ }^{2}$ Médias seguidas de mesma letra na vertical não diferem estatisticamente ao nível de 5\%, pelo teste de Tukey.

Durante os ensaios, as temperaturas do motor estiveram dentro de valores considerados normais: óleo lubrificante, 89 a $112^{\circ} \mathrm{C}$; saída de água do motor, 82 a $85^{\circ} \mathrm{C}$; ar de admissão, 30 a $34^{\circ} \mathrm{C}$; gases de escape, 622 a $638^{\circ} \mathrm{C}$; óleo combustível na entrada da bomba injetora, 32 a $37^{\circ} \mathrm{C}$ (um sistema de refrigeração instalado na bancada, impedia que o óleo ultrapassasse $40^{\circ} \mathrm{C}$ ).

Conforme constata-se no Quadro 2, à queda máxima de potência, observada para B10 e B100, correspondeu um aumento máximo de 7,3\% no consumo específico de combustível com B100. Diferentes valores para a variação de potência e do consumo específico de combustível são observados por outros autores. O uso de metil éster de girassol (B100) por Kaufman \& Ziejewski (1984) em um motor diesel 74,6 kW, 4 cilindros, injeção direta, em testes dinamométricos por 200 horas resultou em potência $5,8 \%$ mais baixa que a obtida com diesel e num aumento de $8,6 \%$, no consumo de combustível. Hilbert et al. (2002), estudando 
misturas de biodiesel em um trator agrícola equipado com motor de $86,0 \mathrm{~kW}$, obteve diferenças de $+1,5 \mathrm{e}-3,7 \%$ na potência da tomada de potência (TDP) para B20 e B100, respectivamente, em relação ao diesel, enquanto que o aumento no consumo específico foi de 6,6 e 13,9\%.

Com as misturas B50 e B100 obtidas a partir do óleo residual, Silva et al. (2004) encontrou reduções médias de 3,4 e 6,3\% para a potência na TDP, em relação ao diesel. O consumo específico, porém, não foi avaliado. Oliveira et al. (2005), estudando as misturas B2, B5, B20 e B100 a partir do óleo de soja degomado, em um trator com potência no motor de 58 $\mathrm{kW}$, concluiram que a potência na TDP com o uso de B100 foi pouco inferior $(-3,25 \%)$ que a apresentada com diesel. Em algumas rotações, as misturas B5 e B100 chegaram a apresentar potência igual ou superior ao diesel. Na avaliação de Maziero et al. (2005), em um motor de $92 \mathrm{kw}$ a redução média na potência foi de 7,6\%, enquanto que o aumento do consumo específico de combustível foi de 9,8\% com B100.

Tem-se verificado nos trabalhos com misturas de biodiesel que a queda de potência nem sempre ocorre linearmente com o aumento da mistura. Nos trabalhos citados, a potência desenvolvida com alguma das misturas intermediárias foi igual a do diesel ou da obtida com B100, o que também ocorreu no presente trabalho (ver Quadro 2) onde a mistura B20 foi levemente superior a de B100.

Os valores de densidade do biodiesel foram superiores ao do diesel e compatíveis com os encontrados na literatura (OLIVEIRA et al., 2005; PETERSON et al., 1999; THOMPSON et al., 1998). Na Figura 1, observa-se o comportamento da densidade, em função da temperatura, para os diferentes combustíveis utilizados que é expresso pelas equações de regressão a seguir.

a) para D: $Y=-1,3362 X+890,31\left(r^{2}=0,9918\right)$

b) para B5: $\mathrm{Y}=-1,2744 \mathrm{X}+891,1\left(\mathrm{r}^{2}=0,9991\right)$

c) para B10: $Y=-1,2943 X+894,0\left(r^{2}=0,9992\right)$

d) para B20: $Y=-1,1176 X+893,6\left(r^{2}=0,9924\right)$

e) para B100: $Y=-1,4392 X+931,2\left(r^{2}=0,9959\right)$

onde:

$\mathrm{Y}=$ densidade do combustível, em $\mathrm{g} / \mathrm{L}$

$\mathrm{X}=$ temperatura do combustível, ${ }^{\circ} \mathrm{C}$.

De acordo com essas equações, a uma temperatura de $40^{\circ} \mathrm{C}$, por exemplo, corresponderiam densidades de 837 , $840,842,849$ e 849 e $874 \mathrm{~g} / \mathrm{L}$, respectivamente para D, B5, B10, B20 e B100.

Quanto ao resultado da análise do óleo lubrificante apresentado no Quadro 3, foram observadas alterações consideradas aceitáveis e que não comprometem o desempenho do motor. O laudo, no entanto, chamava a atenção para o teor de óxido de silício identificado em lâmina de óleo em nível ainda tolerável. As alterações mais expressivas foram relativas à viscosidade, à presença de água e ao teor de ferro.

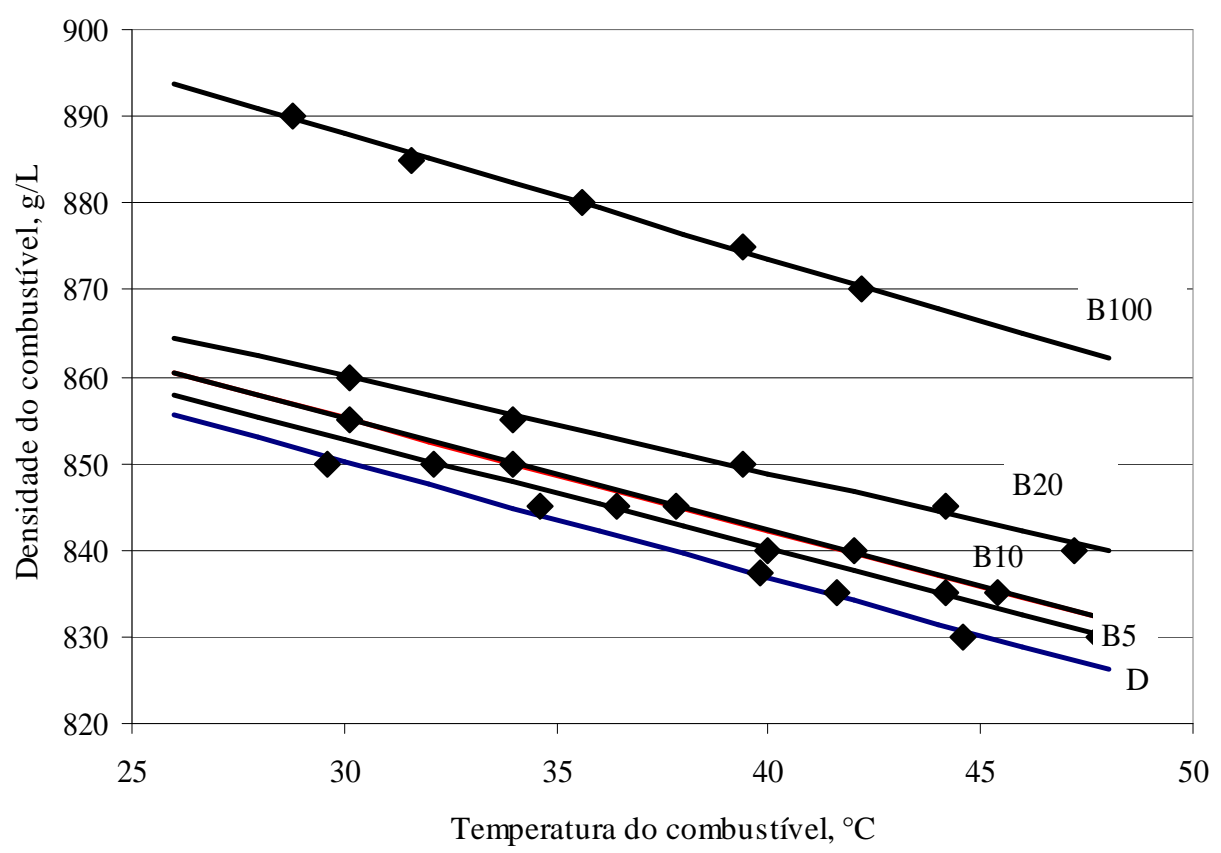

Figura 1 - Densidade em função da temperatura para os combustíveis utilizados. 
Quadro 3-Características do óleo lubrificante.

\begin{tabular}{|lcc|}
\hline \multicolumn{1}{|c}{ Parâmetros avaliados } & Antes dos ensaios & Após 96h de ensaio \\
\hline a) Físico-químicos & & 0,2 \\
Fuligem, \% & 0,1 & 63,04 \\
Viscosidade, c ST 40 & 98,82 & 0,12 \\
Água, \% & 0,06 & 0,0 \\
Diluição, \% & 0,0 & 0,05 \\
Índice de precipitação & Leves traços & 10,4 \\
T.B.N. & 12,6 & \\
b) Teor de minerais & & 0,10 \\
Alumínio & 0,10 & 0,10 \\
Cobre & 0,10 & 0,10 \\
Cromo & 0,10 & 10,8 \\
Ferro & 2,3 & 0,10 \\
Silício & 0,10 & 0,10 \\
Chumbo & 0,10 & normal \\
d) Exame morfológico de partículas (EMP) & & normal \\
Liga Ferr >5 < 15 u & normal & Atenção \\
Fibras plásticas & normal & Traços \\
Óxido de silício & & \\
\hline
\end{tabular}

Os parâmetros físico-químicos admissíveis, para o motor alimentado com óleo diesel, são: máx. 1,5\% para a fuligem (resíduos insolúveis de combustível parcialmente queimado); máx. 3,5\% para a diluição (quantidade de combustível presente no óleo lubrificante), máx. 1,0\% para o índice de precipitação ( presença de componentes insolúveis no óleo); mín. 2,5 mg KOH/g para TBN (número total de basicidade) e máx. de $0,2 \%$ para o teor de água.

Peterson et al. (1999) analisaram a contaminação do óleo lubrificante por metais (alumínio e ferro) em um motor diesel, a intervalos de $100 \mathrm{~h}$ durante 1000 horas de uso com misturas (B25, B50 e B100) de éster etílico de óleo de soja hidrogenada. Não se referindo aos valores encontrados dentro da faixa de normalidade, concluíram apenas que a presença dos metais com o uso de B100 foi igual ou menor que a ocorrida com B25 ou B50. Nos testes de durabilidade realizados por Kaufmann \& Ziejewski (1984) com metil éster de óleo de girassol o consumo de óleo lubrificante foi satisfatório e um pouco de diluição de combustível foi notado.

\section{CONCLUSÕES}

- O uso das misturas B5, B10, B20 e B100 de éster etílico de óleo de girassol proporcionou alteração no desempenho do motor, com redução máxima de $2,2 \%$ na potência e aumento máximo de 7,3\%, no consumo específico.
- A análise do óleo lubrificante, após 96 horas de uso de B100, apresentou valores considerados aceitáveis para motores diesel.

\section{AGRADECIMENTO}

À Fundação de Apoio à Pesquisa do Estado de São Paulo - FAPESP, pelo suporte financeiro ao trabalho (Proc. 02/04492-0).

\section{REFERÊNCIAS BIBLIOGRÁFICAS}

ALI, Y.; HANNA, M. A. Durability testing of a diesel fuel, methyl tallowate and ethanol blend in a Cummins N14-410 diesel engine. Transactions of the ASAE, Saint Joseph, v. 39, n. 3 p. 793-797, 1996.

ALI, Y.; HANNA, M. A.; BORG, J. E. Effect of alternative diesel fuels on heat release curves for Cummins N 14-410 diesel engine. Transactions of the ASAE, Saint Joseph, v. 39, n. 3, p. 407-414, 1996.

ASSOCIAÇÃO BRASILEIRA DE NORMAS TÉCNICAS. NBR 13400: tratores agrícolas: determinação do desempenho na tomada de potência. Rio de Janeiro, 1995. 7 p.

ASSOCIAÇÃO BRASILEIRA DE NORMAS TÉCNICAS. NBR ISO 1585: veículos rodoviários: código de ensaio de motores: potência líquida efetiva. Rio de Janeiro, 1996. 7 p. 
CORREAA, I. M. Desempenho operacional de pneus radiais e diagonais em função da pressão de inflação, da condição de superfície do solo e da condição de acionamento da tração dianteira. 2000. $121 \mathrm{f}$. Tese (Doutorado) Universidade Estadual Paulista, Botucatu, 2000.

HILBERT, J. A.; TESOURO, M. O.; AUCUNÁ, M. O.; PINCU, M. S. Rendimiento comparativo de biodiesel y gasoil em tractores agrícolas. Buenos Aires: INTA, 2002. Disponível em: <http://www.inta.gov.br/iir/info/ documentos/energia/resbiodiesel.htm>. Acesso em: $15 \mathrm{dez}$. 2003.

KAUFMAN, K. R.; ZIEJEWSKI, M. Sunflower methyl esters for direct injected diesel engines. Transaction of the ASAE, Saint Joseph, v. 42, n. 1, p. 1626-1633, 1984.

MAZIERO, J. V. G.; CORRÊA, I. M.; TRIELLI, M. A.; BERNARDI, J. A.; D‘AGOSTINI, M. Avaliação do desempenho de um motor de ignição por compressão utilizando óleo diesel e éster etílico de óleo de girassol como combustível. In: CONGRESSO BRASILEIRO DE PLANTAS OLEAGINOSAS, ÓLEOS, GORDURAS E BIODIESEL, 2., 2005, Varginha, MG. Anais... Lavras: UFLA, 2005. CD-ROM.
OLIVEIRA, E.; SILVA, F. M. da; CONDE, A. do P.; BARBOSA, R. L.; SOUZA, R. G.; LIMA, P. H. G. Desempenho comparativo de motor de combustão alimentado com diesel, B2, B5, B20 e B100. In: CONGRESSO BRASILEIRO DE PLANTAS OLEAGINOSAS, ÓLEOS, GORDURAS E BIODIESEL, 2., 2005, Varginha, MG. Anais... Lavras: UFLA, 2005. CD-ROM.

PETERSON, C. L.; THOMPSON, J. C.; TABERSKI, J. S. One-thousand-hour engine durability test with HySEE and using a $5 \mathrm{X}-\mathrm{MA}$ test cycle. Transaction of the ASAE, Saint Joseph, v. 42, n. 1, p. 23-30, 1999.

SILVA, F. M. da; LOPES, A.; CASTRO NETO, P.; DABDOUB, M. J.; SALVADOR, N.; SILVA, R. P. da. Desempenho do motor de combustão alimentado com diesel, B50 e B100. In: CONGRESSO BRASILEIRO DE PLANTAS OLEAGINOSAS, ÓLEOS, GORDURAS E BIODIESEL, 1., 2004, Varginha, MG. Anais... Disponível em: <http://www.deg.ufla.br/oleo/anais/ artigos/e05.pdf $>$. Acesso em: 15 jun. 2005.

THOMPSON, J. C.; PETERSON, C. L.; REECE, D. L.; BECK, S. M. Two-year storage study with methyl and ethyl esters of rapessed. Transaction of the ASAE, Saint Joseph, v. 41, n. 4, p. 931-939, 1998. 